\title{
Relation of Pancreatic Enzymes and Serum Transaminases Levels with Modified CT Severity Index In Acute Pancreatitis
}

\author{
SHARIF MM ${ }^{\mathrm{a}}$, RAHMAN MT $^{\mathrm{b}}$, FERDOUSI MA $^{\mathrm{c}}$, TAHER MA ${ }^{\mathrm{d}}$, SHEGUFTA F $^{\mathrm{e}}$, AZAM $^{\mathrm{f}}$
}

\begin{abstract}
Introduction: Diagnosis of patients with severe acute pancreatitis as soon as possible is critical for achieving optimal outcomes. Management depends largely on severity. Medical treatment of mild acute pancreatitis is relatively straightforward. Treatment of severe acute pancreatitis involves intensive care. Surgical intervention (open or minimally invasive) is indicated in selected cases.

Methodology: A total number of 59 subjects were enrolled in this present prospective study in the department of Radiology and Imaging, BIRDEM during two years aim to find out the correlation between $C T$ severity index and patient serum enzyme levels in acute pancreatitis. Patients with clinically suspected and biochemically diagnosed acute pancreatitis referred to Radiology and Imaging department from department of gastroenterology (GHPD), BIRDEM for imaging investigation were enrolled in this study. Severity of acute pancreatitis was measured by both clinical and imaging staging (Modified CT severity index) in mild, moderate and severe groups. Subjects clinical information's and serum enzymes were recorded and analyzed by computer software SPSS (Ver. 20-IBM).
\end{abstract}

Result: Mean age of the study subjects was $36.43 \pm 13.85$ years. The age ranged from 20-63 years and the maximum

a. Dr. Md. Mofazzal Sharif, MD (Radiology and Imaging), Consultant, Department of Radiology and Imaging, North Bengal Medical College, Sirajganj.

b. Dr. Md. Towhidur Rahman, MD (Radiology and Imaging), Medical Officer, Department of Radiology and Imaging, BIRDEM, Dhaka

c. Dr. Mahfuz Ara Ferdousi, M. Phil (Radiology and Imaging), Associate Professor, Department of Radiology and Imaging, BIRDEM, Dhaka

d. Dr. Md. Abu Taher, MD (Radiology and Imaging). Associate Professor, Department of Radiology and Imaging, BIRDEM.

e. Dr. Farzana Shegufta, Assistant Professor, Department of Radiology and Imaging, BIRDEM, Dhaka

f. Dr. Golam Azam, MD (Hepatology), Assistant Professor, Department of GHPD, BIRDEM, Dhaka

Address of correspondent: Dr. Md. Mofazzal Sharif, MBBS, MPH (Epidemiology), MCPS \& MD (Radiology and Imaging), Consultant, Department of Radiology and Imaging, North Bengal Medical College, Sirajganj, E-mail: mofazzal.sharif@gmail.com Received: 18 December 2013 Accepted: 20 March 2015 number was found in 21 to 40 years age group. Male female ratio was almost 1.56:1. It was seen that diffuse pancreatic enlargement was noted in $20.33 \%$ subjects. Pancreatic inflammation with and without peripancreatic fat involvement were observed in $22.72 \%$ and $57.62 \%$ subjects respectively. Pseudocyst formation (11.86\%), ascites (23.72\%), renal fascia involvement (37.28\%) and pleural effusion (30.5\%) were seen in CT scan. The results of the interpreter analysis was Kappa $=0.852$ with $p<0.001$ when association of severity by clinical scoring and CT severity scoring was compared and revealed that measure of agreement, while statistically significant, was almost perfect agreement. CT severity index in acute pancreatitis had statistically significant ( $p$ is less than 0.05 ) relation with serum amylase (0.738), lipase (0.638), SGOT (0.581) and SGPT (0.365) during admission which was found on simple linear regression test.

Conclusion: This present study concluded that modified CT severity index correlated well with patient's enzymes level (Amylase, lipase, SGOT, SGPT) as well as clinical findings in acute pancreatitis

Key words: Modified CT severity index, acute pancreatitis, serum enzyme level.

(Birdem Med J 2015; 5(Supplement 1): 18-24)

\section{Introduction}

Pancreatitis is an inflammatory process in which pancreatic enzymes autodigest the gland. The gland sometimes heals without any impairment of function or any morphologic changes; this process is known as acute pancreatitis. ${ }^{1-3}$ Pancreatitis can also recur intermittently, contributing to the functional and morphologic loss of the gland; recurrent attacks are referred to as chronic pancreatitis. Once a working diagnosis of acute pancreatitis is reached, laboratory tests are obtained to support the clinical impression, to help define the etiology, and to look for complications. Diagnostic imaging is unnecessary in most cases but may be obtained when the diagnosis is in doubt, when severe pancreatitis is present, or when a given imaging 
study might provide specific information needed to answer a clinical question. Image-guided aspiration may be useful. Genetic testing may be considered. ${ }^{4-6}$ Management depends largely on severity. Medical treatment of mild acute pancreatitis is relatively straightforward. Treatment of severe acute pancreatitis involves intensive care; the goals of medical management are to provide aggressive supportive care, to decrease inflammation, to limit infection or superinfection, and to identify and treat complications as appropriate. Surgical intervention (open or minimally invasive) is indicated in selected cases. ${ }^{3}$

For predicting severity of acute pancreatitis several scoring system like Ranson's ${ }^{7}$ criteria, the Imrie scoring system, ${ }^{1,2}$ the Acute Physiology and Chronic Health Evaluation System, ${ }^{3}$ Balthazar CT severity index, ${ }^{8}$ Modified CT severity ${ }^{4}$ index were described. Ranson's ${ }^{7}$ included parameters like-age in years, white blood cell count, blood glucose, serum AST, serum LDH and estimation of fluid sequestration. APACHE II and APACHE III systems stated evaluating patient with acute pancreatitis by observing vital functions like CNS, CVS and renal functions. Balthazar ${ }^{8} \mathrm{CT}$ severity index included direct observation of patient outcome and correlated it with contrast enhanced CT scan (CECT scan) describing only pancreatic inflammation and necrosis. It did not include peripancreatic fat abnormalities, fluid collection or extrapancreatic complications (pleural effusion, ascites and GIT complication). The modified CT severity index described both intra and extra pancreatic findings in acute pancreatitis and correlates with patient outcome parameters. ${ }^{4,9}$ Unfortunately, most of the scoring or grading systems had some limitations. Among them modified CT severity index correlated well with patient outcome in acute pancreatitis as well as fulfill the criteria of for an ideal predictor of scoring severity of acute pancreatitis. ${ }^{4}$ Acute pancreatitis is a disease with significant mortality and morbidity. ${ }^{1}$ Almost in every case other than biochemical tests and USG, CT scan are being done to assess the degree of extent of pancreatic necrosis and extrapancreatic involvement. It is important to select an appropriate severity categorization for a disease to take early necessary measures. Thus, this would be a beneficial tool for the doctor to make quick decision about patient as well as for the patient by prompt relief of their suffering.

\section{Methodology}

This prospective study was carried out in the department of Radiology and Imaging, Bangladesh Institute Of Research And Rehabilitation In Diabetes, Endocrine And Metabolic Disorders (BIRDEM) for a period of two year from July, 2011 to June, 2013 with the aim to find out the correlation between $\mathrm{CT}$ severity index and patient serum enzymes level in acute pancreatitis. Patients with clinically suspected and biochemically diagnosed acute pancreatitis referred to Radiology and Imaging department from (Department of Gastro Hepatic Pancreatic Disorders) (GHPD), BIRDEM for imaging investigation were enrolled in this study. Prior to the commencement of this study the research protocol was approved by ethical review committee (Local ethical committee, BADAS). Patients performing contrast enhanced CT scan within 7 days after onset of symptoms were included. Those patients performing non-contrast CT scan or performing contrast enhanced CT scan 7 days after onset of symptoms were excluded from the study. A total number of 59 subjects were included in this study. All the selected subjects underwent contrast enhanced helical CT of abdomen. CT scan was performed on dual slice helical scanner (Siemens, Somatom Emotion Dueo). Demographic information was prospectively recorded and substantiated by means of inspection of medical records. Information included the subject's age, gender and serum enzymes levels. Al the study subjects were followed up to discharge. CT measurement of severity index (Mortele et al, 2004) ${ }^{4}$ of acute pancreatitis was done by researcher at first and findings were confirmed by consultant radiologist of the Department of Radiology and Imaging, BIRDEM who did not know patient's serum enzyme level to eliminate observation bias. Subjects were categorized as mild, moderate and severe by CT severity index. Subject's clinical condition was assessed by Glasgow Prognostic score and categorized mild, moderate and severe classes also. Then agreement between these scoring system was calculated by Kappa test to observe whether CT severity index correlate with clinical condition (By Glasgow Prognostic score) of the subjects. Simple linear regression test was done between CT severity index and subject's serum amylase, lipase, SGOT and SGPT to find out relation between them. Clinical history, laboratory findings of serum enzymes, computed tomographic findings were collected in a pre-designed data collection sheet. 
Glasgow Prognostic scoring systems (For clinical staging)

To predict severe pancreatitis, the Glasgow system (Mortele et al, 2004) 4 is a simple prognostic system that uses 8 factors [white cell count, glucose, urea, $\mathrm{PO}_{2}$, calcium, LDH, transaminases (SGOT, SGPT), albumin] during the first 48 hours following admission for pancreatitis,. A point is assigned if a certain breakpoint is met at any time during that 48 -hour period. The parameters and breakpoints are:

- Serum albumin $<32 \mathrm{~g} / \mathrm{L}(3.2 \mathrm{~g} / \mathrm{dL})=1$ point

- Arterial $\mathrm{PO}_{2}$ on room air $<8 \mathrm{kPa}(60 \mathrm{mmHg})=1$ point

- Serum calcium $<2 \mathrm{mmol} / \mathrm{L}(8 \mathrm{mg} / \mathrm{dL})=1$ point

- Blood glucose $>10.0 \mathrm{mmol} / \mathrm{L}(180 \mathrm{mg} / \mathrm{dL})=1$ point

- Serum $\mathrm{LDH}>600$ unit $/ \mathrm{L}=1$ point

- Serum urea nitrogen $>16.1 \mathrm{mmol} / \mathrm{L}(45 \mathrm{mg} / \mathrm{dL})=1$ point

- $\quad \mathrm{WBC}$ count $>15 \times 10^{\wedge} 9 / \mathrm{L}\left(15 \times 10^{\wedge} 3 / \mathrm{mm}^{3}\right)=1$ point

- Transaminases (SGOT and SGPT) $>200 \mathrm{IU} / \mathrm{L}=1$ point

The addition of the parameter points yielded the Glasgow prognostic criteria. The score ranged from 0 to 8. If the score was $0-2$, the likelihood of mild pancreatitis was high. If the score was 3-4, moderate pancreatitis was likely and if score was 5 and more severe pancreatitis was more likely.

\section{Results}

Age gender distribution of the study subject: A total of 59 patients were included in the study and they were divided into four age groups. Mean age of the study subjects was $36.43 \pm 13.85$ years with male female ratio was almost 1.56:1. The age ranged from $20-63$ years and the maximum number was found in 21 to 40 years age group. The number of male was $36(61.02 \%)$ and female was $23(38.98 \%)$.

CT scan findings of the study subjects: It was seen that diffuse pancreatic enlargement was noted in $20.33 \%$ subjects. Pancreatic inflammation with and without peripancreatic fat involvement were observed in $22.72 \%$ and $57.62 \%$ subjects respectively. Pseudocyst formation $(11.86 \%)$, ascites $(23.72 \%)$, renal fascia involvement (37.28\%) and pleural effusion (30.5\%) were seen in CT scan. Pancreatic necrosis (More than $30 \%$ in $23.72 \%$ and less than $30 \%$ in $27.11 \%$ ) was revealed.

Association of severity between clinical scoring with CT severity index in acute pancreatitis: Severity of acute pancreatitis was evaluated by clinical findings on basis of Glasgow prognostic score and Modified CT severity index. Image analysis for each case was assessed by using the modified CT severity index and categorized as mild ( $0-2$ points), moderate ( $4-6$ points), or severe (8-10 points). Glasgow prognostic score ranged from 0 to 8 . If the score was $0-2$, the likelihood of mild pancreatitis was high. If the score was $3-4$, moderate pancreatitis was likely and if score was 5 and more severe pancreatitis was more likely. The results of the interpreter analysis was Kappa $=0.852$ with $p<0.001$ when association of severity by clinical scoring and CT severity scoring was compared. This measure of agreement, while statistically significant, was almost perfect agreement.

Change of important enzymes in different pancreatitis groups: Serum enzymes levels are shown in Table I and Figure 1.

Relation of CT severity index with patient's serum enzymes at admission: CT severity index in acute pancreatitis had statistically significant ( $p$ is less than 0.05 ) relation with serum amylase $(0.738)$, lipase $(0.638)$, SGOT (0.581) and SGPT (0.365) during admission which was found on simple linear regression test.

\section{Table I}

Change of important enzymes in different pancreatitis groups (Total subjects were 59 where, mild-21, moderate-23 and severe-15)

CT severity groups

Mild

Moderate

Severe

Mild

Moderate

Severe

Mild

Moderate

Severe

Mild

Moderate

Severe
Amylase (mean $\pm \mathrm{SD} ; \mathrm{U} / \mathrm{L}$ ) $300.64 \pm 128.36$ $650.63 \pm 153.88$ $798.97 \pm 311.22$ Lipase (mean $\pm \mathrm{SD}$; U/L) $549.12 \pm 127.64$ $876.81 \pm 372.49$ $1061.59 \pm 584.22$ SGOT (mean $\pm \mathrm{SD}$; U/L) $50.47 \pm 6.36$ $53.64 \pm 14.98$ $58.29 \pm 20.67$ SGPT (mean $\pm \mathrm{SD}$; U/L) $44.11 \pm 20.39$ $51.63 \pm 29.67$ $65.15 \pm 42.90$ 


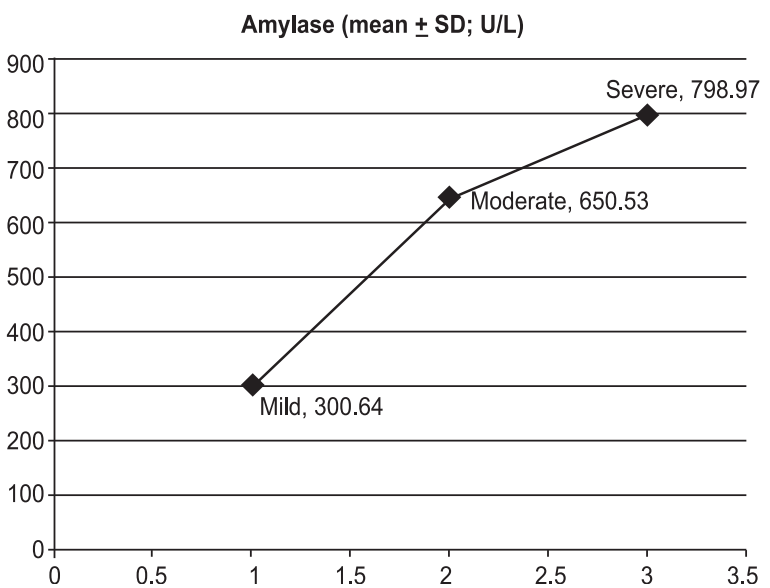

Line diagram showing serum amylase in different pancreatitis groups.

SGOT (mean \pm SD; U/L)

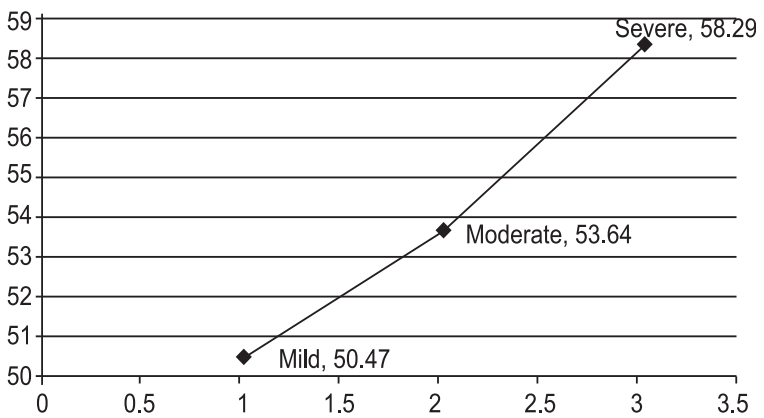

Line diagram showing serum SGOT levels in different pancreatitis groups.

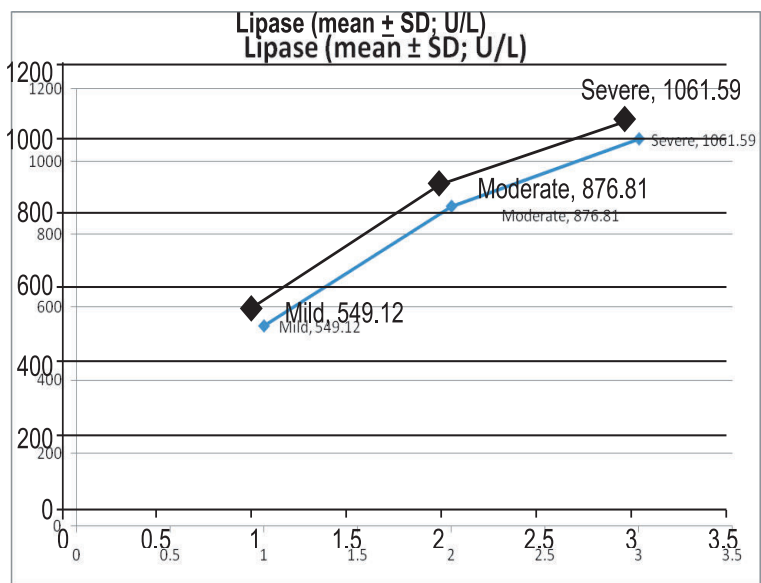

Line diagram showing serum lipase levels in different groups of pancreatitis.

SGPT (mean \pm SD; U/L)

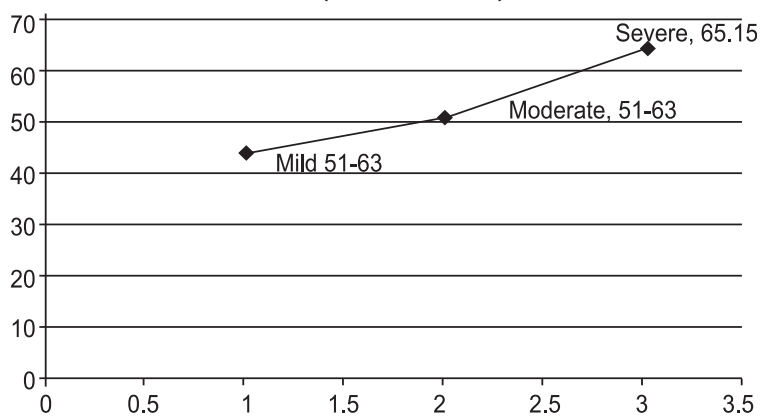

Line diagram showing serum SGPT levels in different pancreatitis groups.

Figure 1: Line diagram showing changes of serum enzymes and transaminases in acute pancreatitis

Illustrations of Imaging findings in different acute pancreatitis with pancreatic enzymes and serum transaminases levels

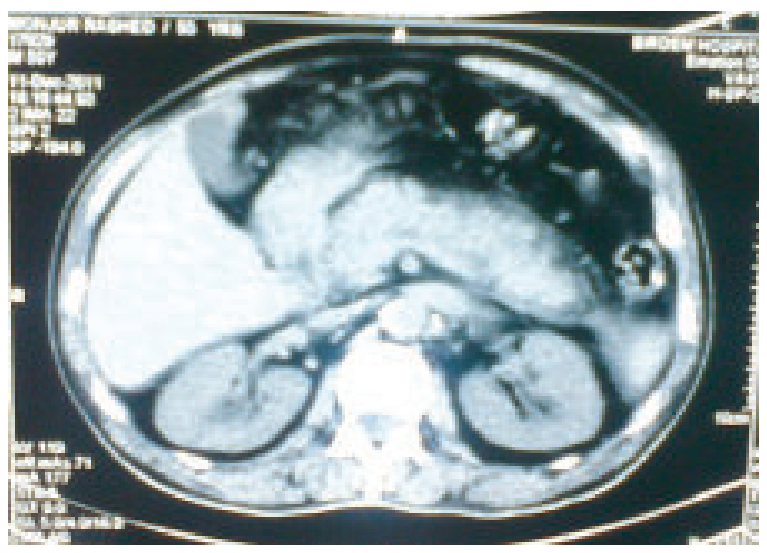

Illustration-I: Contrast enhanced CT scan showing mild intrinsic pancreatic inflammation with peripancreatic fat inflammation ( $=2$ points). This scan was taken from 55 years old male patient with clinically suspected acute pancreatitis was scored as 2 (Mild acute) according to midified CT severity index. The subject had serum amylase level of $287 \mathrm{U} / \mathrm{L}$, lipase level of $456 \mathrm{U} / \mathrm{L}$, SGOT level of $44 \mathrm{U} / \mathrm{L}$ and SGPT level of $39 \mathrm{U} / \mathrm{L}$. Subject had complete remission within 4 days without any complecation. 


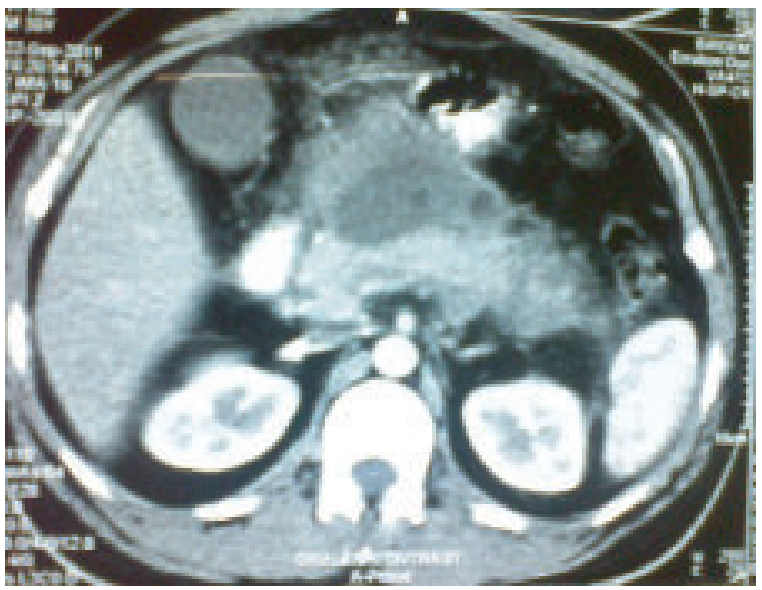

Illustration-II: Contrast enhanced CT scan showing intrinsic pancreatic inflammation with peripancreatic fat inflammation ( $=2$ points), pancreaticnecrosis $<30 \%$ ( $=2$ points).This CT image was taken from 30 years old female subject of with clinically suspected case of acute pancreatitis with elevalted serum amylase level scored as 4 (Moderate acute pancreatitis) on modified CT severity index. was scored as 2 according to midified CT severity index. The subject had serum amylase level of $637 \mathrm{U} / \mathrm{L}$, lipase level of $819 \mathrm{U} / \mathrm{L}$, SGOT level of $47 \mathrm{U} /$ $\mathrm{L}$ and SGPT level of $42 \mathrm{U} / \mathrm{L}$. Subject had shock on admission and stayed in the hospital for 7 days.

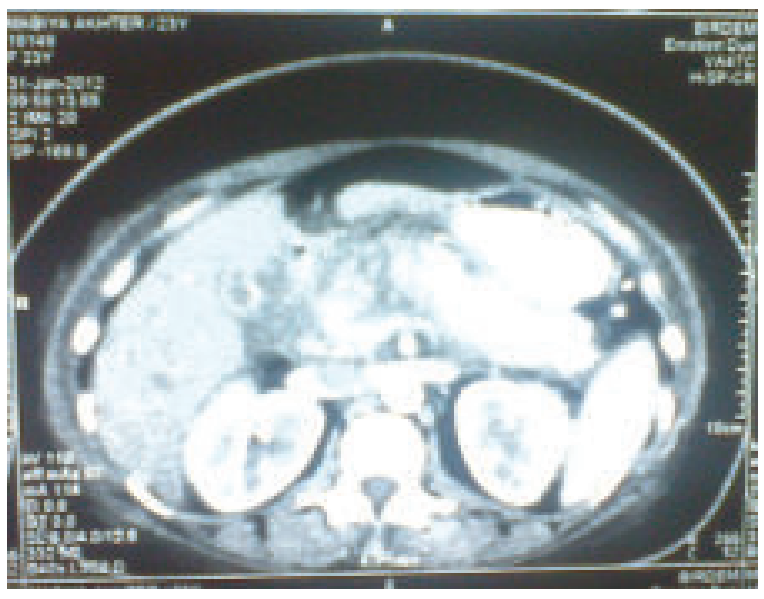

Illustration-III: Contrast enhanced CT scan showing pancreatic inflammation ( $=2$ points), pancreaticnecrosis $>30 \%$ (=4 points) and left sided pleural effusion ( $=2$ points).

This CT image was taken from 23 years old female subject who was admitted with shock and respiratory failure. She was scored as 8 (Severe acute pancreatitis) on modified CT severity index. The subject had serum amylase level of $588 \mathrm{U} / \mathrm{L}$, lipase level of $902 \mathrm{U} / \mathrm{L}$, SGOT level of $53 \mathrm{U} / \mathrm{L}$ and SGPT level of $48 \mathrm{U} / \mathrm{L}$. Subject stayed in the hospital for 11 days.

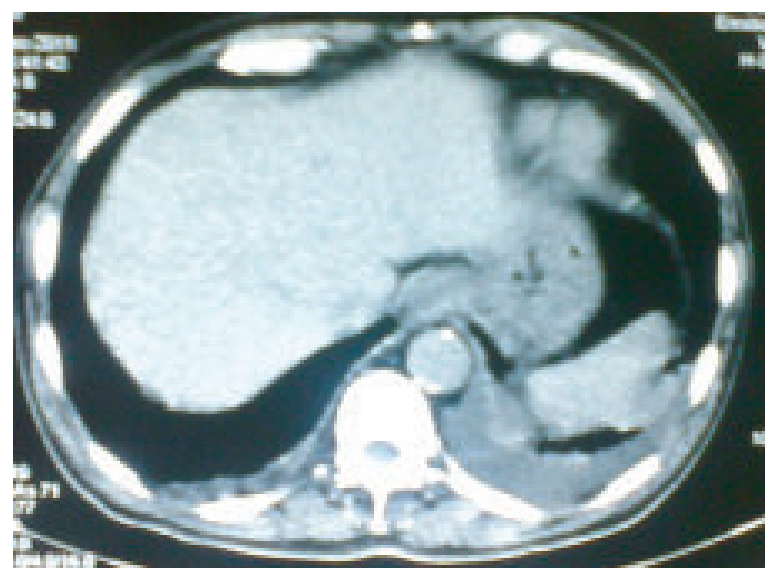

\section{Discussion}

Acute pancreatitis generally affected all gender groups especially male were more affected than female (David et al, 2006). Whitcomb et al (2006) reported that although median age at onset depends on the etiology majority of the subjects with acute pancreatitis ranged from 2575 years. Mean age of the study subjects was $36.43 \pm$ 13.85 years. The age ranged from $20-63$ years and the maximum number was found in 21 to 40 years age group. The number of male was $36(61.02 \%)$ and female was 23 (38.98\%). Male female ratio was almost 1.56:1. Otsuki et al (2006) found that about $20 \%$ of the acute pancreatitis was severe with a mortality of about $20 \%$. Necrosis would be followed by a systemic inflammatory response syndrome and organ failure. The further clinical course was then determined by bacterial infection. Otsukiet et al (2006) also found that mild pancreatitis; the CT features were ranged from a normal-appearing pancreas with no peripancreatic abnormalities to diffuse enlargement and heterogeneous attenuation of the gland with illdefinition of the border. In severe pancreatitis, there is, in addition to the features stated above, a lack of normal enhancement of part of the pancreas or the entire pancreas, in keeping with necrosis. Area as of nonenhancement was considered a reliable CT sign for necrosis. Other characteristics of severe pancreatitis included more extensive peripancreatic inflammatory change than was seen in mild acute pancreatitis. Usually, this was associated with focal fluid collections. Other findings in CT scan were presence of pseudocyst, 
ascites, pleural effusion etc. In present study, it was seen that diffuse pancreatic enlargement was noted in $20.33 \%$ subjects. Pancreatic inflammation with and without peripancreatic fat involvement were observed in $22.72 \%$ and $57.62 \%$ subjects respectively. Pseudocyst formation $(11.86 \%)$, ascites $(23.72 \%)$, renal fascia involvement (37.28\%) and pleural effusion (30.5\%) were seen in CT scan. Pancreatic necrosis (More than $30 \%$ in $23.72 \%$ and less than $30 \%$ in $27.11 \%$ ) was revealed.

Severity of acute pancreatitis was evaluated by clinical findings and CT severity index. The results of the interpreter analysis was Kappa $=0.852$ with $\mathrm{p}<0.001$. This measure of agreement, while statistically significant, was almost perfect agreement. This result reflected that modified CT severity index had relation with clinical findings. Similar study result was observed in the study conducted by Bollen et al (2006) where it was reported that there were no statistically significant differences between the predictive accuracies of CT and clinical scoring systems and was concluded that the predictive accuracy of CT scoring systems for severity of AP was similar to clinical scoring systems. Previous studies ${ }^{5,6}$ was conducted to prove modified CT severity index as ideal prognostic method that allowed differentiation between patients with mild and those with severe pancreatitis should be accurate, easy to use, and widely available and should have low interobserver variability. Other studies was conducted to validate modified CT severity index to be applicable for early diagnosis in the disease process, so that patients who could potentially develop complications can be monitored more closely or empirically treated, for example with fluid resuscitation. ${ }^{5}$ Since 1974, several clinical and radiologic scoring systems have been developed for this purpose, including Ranson's criteria, ${ }^{6}$ the acute physiology and chronic health evaluation (APACHE II) scoring system, ${ }^{7}$ and the CT severity index. The CT severity index, developed by Balthazar and colleagues in $1994^{8}$ was a significant advance because it helped clinicians to discriminate among mild, moderate, and severe forms of pancreatitis. Although this system had been successfully used to predict overall morbidity and mortality in patients with acute pancreatitis, it had limitations like the score obtained with the index did not significantly correlate with the subsequent development of organ failure and extrapancreatic parenchymal complications or peripancreatic vascular complications.
On the other hand, this present modified CT severity index correlated well patient clinical outcome. ${ }^{3,} 4$ As stated above all the previous studies were carried out with retrospective study design concerning only clinical outcome and imaging findings. In present prospective study it was seen that there was marked increase of serum amylase, lipase, SGOT and SGPT in mild to severe acute pancreatitis which was determined by modified CT severity index in imaging findings basis. CT severity index in acute pancreatitis had statically significant ( $p$ is less than 0.05$)$ relation with serum amylase $(0.738)$, lipase (0.638), SGOT (0.581) and SGPT (0.365) during admission. It was also observed that there was marked reduction of serum amylase, lipase, SGOT and SGPT in mild pancreatitis and all the markers were almost came to base line during discharge in modified CT diagnosed mild group. During discharge, reductions of various markers were less in moderate pancreatitis. In severe pancreatitis serum amylase, lipase, SGOT and SGPT levels remained almost unchanged during discharge comparing to the level at admission. There was significant change of serum enzymes levels reduction at discharge compared to admission levels.

\section{Conclusion}

This present study concluded that modified CT severity index correlated well with patient's enzymes level (amylase, lipase, SGOT, SGPT) as well as clinical findings in acute pancreatitis. It could be also said that from imaging findings diagnosis of acute pancreatitis could be made along with its categorization in acute ill subjects with pancreatitis when clinical diagnosis is difficult and serum enzymes levels are not available.

\section{Acknowledgement}

It is our great honor to work with department of GHPD, BIRDEM. We would like to show profound gratitude to the respected senior teacher, doctors and post-graduate students who helped us for doing this research work. We express our thanks and indebtedness all the patients who voluntarily accepted to be the study subjects.

\section{References}

1. Telem DA, Bowman K, Hwang J, Chin EH, Nguyen SQ, Divino CM. Selective management of patients with acute biliary pancreatitis. J Gastrointest Surg 2009; 13 (12): 2183-88.

2. Whitcomb DC, Yadav D, Adam S. Multicenter approach to recurrent acute and chronic pancreatitis in the United 
States: the North American Pancreatitis Study 2 (NAPS2). Pancreatology 2008; 8(5): 520-31.

3. Li H, Qian Z, Liu Z, Liu X, Han X, Kang H. Risk factors and outcome of acute renal failure in patients with severe acute pancreatitis. J Crit Care 2010; 25 (2): 225-29.

4. Mortele KJ, Wiesner W, Intriere L, Shankar S, Zou KH, Kalantari BN, Perez A, vanSonnenberg E, Ros PR, Banks PA, Silverman SG. A modified CT severity index for evaluating acute pancreatitis: improved correlation with patient outcome. AJR Am J Roentgenol. 2004; 183(5): 1261-65.

5. Bollen TL, Santvoort HCV, Besselink MG, Leeuwen MSV, Horvath KD, Freeny PC, Gooszen HG. The Atlanta
Classification of acute pancreatitis revisited. Pancreas 2006; 33: 448-49.

6. Agarwal N. Assessment of severity in acute pancreatitis. Am J Gastroenterol 1991; 86:1385-91.

7. Ranson JHC, Rifkind KM, Roses DF, Fink SD, Eng K, Spencer FC. Prognostic signs and the role of operative management in acute pancreatitis. Surg Gynecol Obstet 1974; 139: 69-81.

8. Knaus WA, Draper EA, Wagner DP, Zimmerman JE. APACHE II: a severity of disease classification system. Crit Care Med 1985; 13: 818-29.

9. Balthazar EJ, Freeny PC, vanSonnenberg E. Imaging and intervention in acute pancreatitis. Radiology 1994; 193: 297-306. 